Instituto Internacional de Investigación y Desarrollo Tecnológico Educativo INDTEC, C.A.

DOI: https://doi.org/10.29394/scientific.issn.2542-2987.2017.2.4.11.186-203

OAI-PMH: http://www.indteca.com/ojs/index.php/Revista Scientific/oai

\title{
Estrategias Pedagógicas para la Conservación de Quebrada la Ciénaga del Municipio Cruz Paredes Estado Barinas 2016
}

\author{
Autor: Jesús Manuel Torres Martínez \\ Universidad Pedagógica Experimental Libertador, UPEL \\ jesm14@hotmail.com \\ Barinas, Venezuela
}

\section{Resumen}

La investigación tiene como propósito la elaboración de estrategias pedagógicas de conservación de la quebrada "la ciénaga" dirigidas a sensibilizar a los habitantes del sector "El centro", Parroquia Barrancas, Municipio Cruz Paredes, Estado Barinas; fue de tipo descriptivo, apoyado en la investigación de campo y fundamentado en un proyecto factible; Las Técnicas e Instrumentos de Recolección de Información se utilizó el Cuestionario, con escala Likert; la Validez y Confiabilidad a través del Juicio de Expertos- Alpha de Cronbach. La población fue de mil seiscientos diez (1610) habitantes del sector el centro; de los cuales se tomó una muestra simple aleatoria al azar de quince (15) personas. Esta información permitió identificar que algunos habitantes del sector poseen conocimientos de que esta naciente de agua es un recurso natural que sostiene una biodiversidad de flora y fauna única del municipio; una porcentaje de las personas posee conocimientos de que la tala y la quema en zonas cercanas a la cuenca de la quebrada son perjudiciales para la vida de este ecosistema; con todo esto es recomendable aplicar estrategias pedagógicas de conservación ambiental en los habitantes del sector el centro para que fortalezcan los valores de conservación con el fin de involucrarlos en la solución de sus problemas.

Palabras clave: estrategias pedagógicas; conservación; participación social. 


\title{
Teaching Strategies for the Conservation of the Cruz Paredes Municipality State Swamp Creek Barinas 2016
}

\begin{abstract}
Research aims at teaching strategies for the conservation of the Gorge "the swamp" aimed to raise awareness among the inhabitants of "The Center", parish Barrancas, Cruz Paredes municipality, Barinas State; was of type descriptive, supported in the research of field and informed in a project feasible; Techniques and information collection instruments used the questionnaire with Likert scale; the validity and reliability on the judgement of experts-Alpha of Cronbach. The population was of thousand six hundred ten (1610) inhabitants of the sector the Center; of which it took a shows simple random to the random of fifteen (15) people. This information allowed us to identify some of the inhabitants of the sector to have knowledge that this source of water is a natural resource that sustains a biodiversity of flora and fauna that are unique of the municipality; a percentage of people has knowledge that the felling and burning in areas near the basin of the ravine are harmful to the life of this ecosystem; with all this is recommended apply strategies pedagogical of conservation environmental in them inhabitants of the sector the Center to strengthen the values of conservation to involve them in the solution of their problems.
\end{abstract}

Keywords: pedagogical strategies; conservation; social participation. 


\section{Introducción}

La educación ambiental es un tema de gran relevancia mundial por cuanto la misma propone educar al hombre para la convivencia armoniosa con el entorno que lo rodea es por este motivo que el agua es el líquido más preciado por la humanidad ya que es vital para la biodiversidad, a medida que transcurre el tiempo ha crecido la población humana; generando una problemática mundial de distribución uso y consumo masivo del agua creando con esto focos de contaminación en su crecimiento intentando cubrir las necesidades humanas sin lograr mantener un equilibrio ecológico.

Todo esto sumado a la destrucción del ambiente, la deforestación, contaminación de lagos, ríos, océanos, pantanos, caños, quebradas, manglares entre otros; sin darse cuenta que el impacto ambiental afectara de forma significativa a las futuras generaciones debido a los intensos cambios climáticos que ya están siendo manifestados por la naturaleza perjudicando la subsistencia de los seres vivos.

En tal sentido la preservación del agua no se debe postergar debido a que su uso es más intenso y necesario a medida que las poblaciones se establecen porque de ella depende el éxito del progreso de las civilizaciones crecientes, en los países latinoamericanos el tema del agua debe ser evaluado significativamente porque la población también está creciendo y el recurso hídrico se está agotando a pesar de su importancia el agua es uno de los recursos más deficientemente administrados en el planeta tierra.

Los seres humanos se preocupan muy poco de hacer disponible y aprovechable este recurso impulsando el menor desperdicio y contaminación. En Venezuela se necesita de un nuevo paradigma que coloque a los ciudadanos en el centro del desarrollo que considere el crecimiento económico como un medio y no como un fin, protegiendo e impulsando las oportunidades de vida preservando las futuras generaciones al igual que la de las actuales 
respetando así los sistemas naturales de los que dependen todos los seres vivos.

Lo anterior descrito es evidente en el estado Barinas, donde también se están administrando mal sus recursos hídricos ya que no están aplicando estrategias que ayuden a conservar los ríos y quebradas que surten de agua a la población en general en consecuencia el Municipio Cruz Paredes no escapa de esta realidad ya que no existen brigadas u organismo que se dediquen a educar a los pobladores, principalmente a las habitantes que están haciendo vida cerca de esta quebrada llamada "la ciénaga". En el marco de la conservación del ambiente se hace necesario la sensibilización de las personas para que sean capaces de abordar y solucionar sus problemas ambientales.

Dentro de este orden de ideas a medidas que la población va en aumento se van ocupando territorios y espacios para la construcción de vivienda sin importar su ubicación y por ende se han dado la tarea de construir cerca de las nacientes de agua y a lo largo de su cauce lo que ha traído como consecuencia la deforestación y contaminación de los suelos por desechos y residuos sólidos orgánicos e inorgánicos como también desembocaduras de aguas negras (lavaplatos, de ropa) entre otros.

Esto ocurre mayormente por no existir una conciencia ambiental que permita a sus pobladores adoptar medidas que permitan mejorar los malos hábitos y poner en práctica un sistema de conservación del cauce de la quebrada la "ciénaga" para el uso y goce de futuras generaciones, en consecuencia, es importante generar desde el seno de la familia los valores y el sentido de pertenencia para poder lograr proteger la quebrada la "ciénaga", la cual es un reservorio de agua formando en riachuelo denominada quebrada la Ciénaga la cual pasa por el corazón del pueblo y desaparece filtrándose en el suelo en las sabanas correspondientes al sector los comunales dado el alto nivel freático en esta área. 
HIDROANDES, la compañía encargada de la explotación y aprovechamiento de la misma construyo los principales pozos de agua que suministrará de agua potable a la población de aproximadamente 40.000 habitantes además es considerado como el pulmón principal de la población Barranqueña.

Es de allí la importancia de establecer acciones o proyectos para el rescate integral de la quebrada. Por lo tanto, se desarrollarán, estrategias y herramientas educativas, que generen concienciación, educación en el cuido y convivencia con los recursos naturales del Municipio. Ubicada en sector el centro, Parroquia Barrancas Municipio Cruz Paredes, estado Barinas. "La ciénaga" es una importante fuente de vida con un gran ecosistema y una variedad incomparable de flora y fauna silvestre; es necesario lograr una interacción más equilibrada con los elementos de la naturaleza, es tener la certeza de lograr mantener un respeto por lo que representa este hermoso afluente y los beneficios que otorga a la población en general.

De aquí se sostiene que la puesta en práctica de estrategias pedagógicas dirigidas a la conservación de la quebrada "la ciénaga" con la comunidad será beneficiosas para todos en general; porqué serán ellos los cuentadantes, los contralores de que se cumplan las exigencias legales con respecto a los infractores del ambiente. De todos aquellos que no saben el daño que ocasionan al talar, quemar y arrojar desperdicios sólidos a la quebrada. Por esto se considera que la educación ambiental debe darse efectivamente a través de estrategias pedagógicas de sensibilización en la conservación de la quebrada "la ciénaga".

Todo esto representará el despertar del interés por la cultura ambiental es por este motivo que la investigación fue de tipo descriptivo, apoyado en la investigación de campo y fundamentado en un proyecto factible, en el desarrollo de la presente investigación buscara a contribuir en la sensibilizar de las personas del sector el Centro Municipio Cruz Paredes, parroquia 
Barrancas del estado Barinas en la conservación y preservación de la quebrada la ciénaga.

La crisis ambiental que se está viviendo hoy en día a despertado una alerta a la sociedad mundial generando gran preocupación y malestar en las personas, ya que todo ha sido producto de la mano destructiva del hombre simplemente por saciar su sed de poder, desarrollo, modernismo; contribuyendo así con el impacto negativo que está generando en el mundo la contaminación y destrucción de recursos naturales el cual está conllevando a los cambios climáticos, como también la desaparición de grandes cuencas naturales, especies animales y vegetales.

Portal situación distintos organismo gubernamentales y no gubernamentales están tomando carta en el asunto para la preservación y conservación del ambiente con sus recursos naturales, el agua como fuente vital para la existencia del ser humano; la quebrada ciénaga ubicada en Barrancas Municipio Cruz Paredes del Estado Barinas está sufriendo esta problemática causada por la contaminación, hoy en día la deforestación la tala y quema de los árboles y vegetación presente a orillas de la naciente de agua, como también el crecimiento urbano han generado disminución del nivel del agua, la perdida de la biodiversidad y su contaminación. Como también el daño a la flora y fauna existente.

Es importante destacar que todo lo planteado ha concebido una serie de interrogante las cuales se enuncia las siguientes:

¿Cuáles son los conocimientos, necesidades y actitudes que poseen los habitantes del sector el centro de la Parroquia Barrancas del Municipio Cruz Paredes?

¿Cuál es la factibilidad económica, social, y educativa de un plan de estrategias pedagógicas para brindar las herramientas al sector el centro de la Parroquia Barrancas del Municipio Cruz Paredes? 
¿Cómo es el diseño de un conjunto de estrategias pedagógicas para los habitantes del sector el centro de la Parroquia Barrancas del Municipio Cruz Paredes?

¿Cómo es la aplicación de las estrategias pedagógicas para proporcionarlas al sector el centro de la Parroquia Barrancas del Municipio Cruz Paredes?

¿Qué valor le da el habitante a la conservación del agua, quebradas o riachuelos?

Estos planteamientos, serán vislumbrados a la situación real de la comunidad.

\subsection{Objetivo de la Investigación}

Implementar estrategias pedagógicas para la conservación de la quebrada La Ciénaga, Sector El Centro, Parroquia Barrancas Municipio Cruz Paredes, Estado Barinas año 2016.

\section{Teoría y Conceptos}

\subsection{Antecedentes de la Investigación}

Gabriela C. (2010), en su trabajo de grado de Maestría Educación Ambiental UNELLEZ en el municipio Guanare, estado Portuguesa realizó una investigación sobre Estrategias Pedagógicas y de Sensibilización en la Educación Ambiental el cual plantió Fomentar Estrategias Pedagógicas y de Sensibilización para la Educación Ambiental en la Escuela Técnica Agropecuaria El Algarrobo, Comprender la situación educativa ambiental de la ETA El Algarrobo, el cual desarrolló por medio de objetivos trazado como Propiciar manejos ambientales, Educación Ambiental hacia el desarrollo sustentable, experiencias Interpretar el grado de sensibilización de los actores.

Este trabajo tuvo como finalidad en fomentar la educación ambiental mediante el uso del manejo como estrategias de Enseñanza aprendizaje, 
mediante proceso que ayuden a vincular los procesos de enseñanzas para resolver problemas ambientales a través de una participación activa, buscando en generar un impacto en la sociedad de manera que sensibilicemos a las personas dándoles impresiones positivas en el cuido del ambiente para tener una mejor calidad de vida.

Dagnubys J. (2009), en su trabajo de grado de Especialización en Educación Comunitaria Universidad Pedagógica Experimental Libertador en él, estado Barinas efectúo el estudio sobre Estrategia de Intervención Comunitaria para la Conservación y Mantenimiento del Rio Santa Bárbara, donde desarrollo objetivos trazados en la Aplicación Estrategia de Intervención Comunitaria para la Conservación y Mantenimiento del Rio Santa Bárbara del año 2008 - 2009.

Dicho trabajo tubo como finalidad la aplicación de estrategias para el mantenimiento y conservación del Rio Santa Bárbara como alternativa para la disminución del daño ambiental en el sector el Rio donde estaría dirigida a los pobladores del sector en busca de sensibilizar a las personas las Cuale sirvieran de multiplicadores para que la población en general del municipio Ezequiel Zamora forme parte activa de la campaña de conservación del Rio Santa Bárbara. Y así se logre un bienestar mutuo donde garantice a las generaciones futuras un ambiente sano y un recurso natural tan importante como el Agua.

\subsection{Definición ambiente}

El ambiente es un conjunto de subsistemas que forman parte de un sistema del planeta tierra entre uno de ellos tendremos los agentes Bióticos y Abióticos que forma junto la biodiversidad del ambiente, tal como lo señala la Ley Orgánica del Ambiente en su Artículo 3.

A los efectos de la presente Ley, se entenderá por: Ambiente: Conjunto o sistema de elementos de naturaleza física, química, 
biológica o socio cultural, en constante dinámica por la acción humana o natural, que rige y condiciona la existencia de los seres humanos y demás organismos vivos, que interactúan permanentemente en un espacio y tiempo determinado.

Se puede decir que el ambiente no es solo lo que rodea el hombre si no es todo lo tangible e intangible que está presente en la tierra y que por ende se debe tener conocimiento del mismo ya que se podrá gozar de ella sin ocasionar ninguna alteración física química que ponga en riesgo la salud de las personas como la naturaleza.

\subsection{Conservación}

Aunque la idea de conservar es probablemente tan antigua como la especie humana, el uso de ese término en el contexto presente es relativamente reciente, a través de los años la conservación ha adquirido muchas connotaciones: para algunos significa la protección de la naturaleza salvaje, para otros el sostenimiento productivo de materiales provenientes de los recursos de la Tierra (Hernandez, 2011a, pág. 16).

La definición más extendidamente aceptada fue presentada en 1980 por la Unión Internacional para la Conservación de la Naturaleza y Recursos Naturales (UICN) como: "La utilización humana de la biosfera para que rinda el máximo beneficio sostenible, a la vez que mantiene el potencial necesario para las aspiraciones de futuras generaciones". (Hernandez, 2011b, pág. 16).

De lo ante expuesto es necesario señalar que la conservación y la preservación de la Biodiversidad existente en el planeta es esencial para garantizar el futuro a nuevas generaciones como también el disfrute de áreas libres de cualquier agente contaminante que pueda ocasionarle daño directo $o$ indirectamente a la población. 


\subsection{Estrategias formativas}

Para la definición o conceptualización sobre la estrategia formativas existen muchas documentaciones, entre la más acertada y correspondiente tenemos la de Cano (2007):

El desarrollo de una competencia es una actividad cognitiva compleja que exige a la persona establecer relaciones entre la práctica y la teoría; transferir el aprendizaje a diferentes situaciones, aprender a aprender, plantear y resolver problemas y actuar de manera inteligente y crítica en una situación (pág. 39).

El autor señala que para realizar las cosas tenemos que tener la base teórica todo lo conceptual los procedimientos las herramientas las técnicas que vamos a emplear para el abordaje de la situación o el problema y posteriormente llevar todo lo plasmado a lo real a la práctica para así lograr la eficacia de los objetivos planteados.

\subsection{Pedagogía ambiental}

La Pedagogía ambiental analiza la influencia provocada por el contacto de la persona con el ambiente cuando dicho contacto no es sistemáticamente educativo y la manera de dirigirlos, parte de una consideración dinámica de la personalidad, y responde a una concepción de la pedagogía, que tiene en cuenta la finalidad o dirección de cada uno de los dos polos constituido del par persona-ambiente, así como también la posición del hombre ante los influjos recibidos a pedagogía ambiental es distinta a la pedagogía escolar ya que se relaciona Profesor alumno y familiar Padre e hijos. (León A, 1998, pág. 521).

Sin embargo la pedagogía ambiental se argumenta donde todo ser humano vive en un ambiente donde existen elementos creados con finalidades no directamente formativas económicas, políticas, sociales que ejerce un modo incidental presiones más o menos más fuerte sobre él, con referencia a esta hoy en día se busca enlazar la pedagogía ambiental con el resto de las 
pedagogías donde se pueda educar a las personas sobre el cuidado del ambiente y el respeto por ella desde la escuela hasta su crecimiento personal y así obtener un ciudadano con valores ambientales.

\section{Metodología}

La metodología a usar comprende la manera que el investigador realizara la sustentación teórica como legales como también recolección y sistematización de los datos obtenidos. Para Tamayo y Tamayo (2003), define al marco metodológico como "Un proceso que, mediante el método científico, procura obtener información relevante para entender, verificar, corregir o aplicar el conocimiento", dicho conocimiento se adquiere para relacionarlo con las hipótesis presentadas ante los problemas planteados". (pág. 37).

Por tal motivo es necesario elaborar una serie de Fases sobre la Investigación que ayudara a desarrollar la manera en la que se abordar el problema las culés se enuncia de la siguiente manera:

Fase I. Diagnóstico Del Problema.

Fase II. Establecer.

Fase III. Diseñar.

Fase IV. Aplicar.

Fase V. Evaluar.

Fase I. Diagnosticar los conocimientos, necesidades y actitudes que poseen los habitantes del sector El Centro de la Parroquia Barrancas Municipio Cruz Paredes, Estado Barinas en el ámbito de conservación y preservación de la quebrada denominada la ciénaga en la comunidad de Barrancas del municipio cruz paredes, estado Barinas año 2016.

Fase II. Establecer las teorías y enfoques que orienten en la conservación ambiental.

Fase III. Diseñar un conjunto de estrategias pedagógicas con el objeto 
de proporcionarles a los habitantes del sector el centro de la Parroquia Barrancas Municipio Cruz Paredes, Estado Barinas las herramientas necesarias para la conservación y preservación de la quebrada la Ciénaga.

Fase IV. Aplicar las estrategias pedagógicas para proporcionarles a los habitantes del sector el centro los conocimientos y actitudes para el abordaje de la problemática ambiental, que está ocurriendo en la quebrada la ciénaga parroquia Barrancas municipio Cruz Paredes.

Fase V. Evaluar la aplicación del plan de estrategias pedagógicas para proporcionarles a los participantes del sector el centro de la Parroquia Barrancas Municipio Cruz Paredes, Estado Barinas

Estas fases ayudaran a desarrollar y entender la problemática del fenómeno y así dará a conocer, mediante que procedimiento y técnicas podremos abordar la problemática, por otra parte, tenemos que la investigación será con un enfoque cuantitativo.

Mayntz et (1980: 198), define el análisis de contenido como "una técnica de investigación que identifica y describe de una manera objetiva y sistemática las propiedades lingüísticas de un texto con la finalidad de obtener conclusiones sobre las propiedades no-lingüísticas de las personas y los agregados sociales. En este sentido, el «texto» puede ser tanto un escrito como un «discurso» oral (y registrado, por ejemplo, en una cinta magnetofónica). Sin embargo, en un sentido más amplio, el análisis de contenido se puede aplicar también a materiales que no sean puramente lingüísticos; por ejemplo, películas o anuncios publicitarios".

En la Siguiente investigación se empleará la técnica de la encuesta y la observación directa, explicando así Hurtado (2010), "tiene aplicación en aquellos problemas que se pueden investigar por método de observación, análisis de fuentes documentales y demás sistemas de conocimiento, además permite el conocimiento de las actitudes, opiniones de los individuos con 
relación al objeto de investigación" (pág. 106).

Se realizarán un cuestionario de ítems con escala Likert Sampieri (2010). Este método fue desarrollado por Rensis Likert en 1932; sin embargo, se trata de un enfoque vigente y bastante popularizado que consiste en un conjunto de ítems presentados en forma de afirmaciones o juicios, ante los cuales se pide la reacción de los participantes, es decir, se presenta cada afirmación y se solicita al sujeto que externe su reacción eligiendo uno de los cinco puntos o categorías de la escala.

A cada punto se le asigna un valor numérico. Así, el participante obtiene una puntuación respecto de la afirmación y al final su puntuación total, sumando las puntuaciones obtenidas en relación con todas las afirmaciones. (pág. 245).

La escala de Likert mide actitudes o predisposiciones individuales en contextos sociales particulares. Se le conoce como escala sumada debido a que la puntuación de cada unidad de análisis se obtiene mediante la sumatoria de las respuestas obtenidas en cada ítem. Por otra parte, se dice que La escala se construye en función de una serie de ítems que reflejan una actitud positiva o negativa acerca de un estímulo o referente. Cada ítem está estructurado con cinco alternativas de respuesta:

( ) Totalmente de acuerdo.

( ) De acuerdo.

( ) Indiferente.

( ) En desacuerdo.

( ) Totalmente en desacuerdo.

Este instrumento servirá para que las personas a encuestar sean libres de expresarse con formulaciones de respuesta libre y expresiva sin que tenga un juicio cerrado en el abordaje de esta problemática que está carrereando a los habitantes del sector el Centro del Municipio Cruz Paredes. Con una La 
muestra, para el presente estudio, sería una muestra intencionada. Al respecto, se seleccionará una muestra intencionada de (10) hogares, para la cual se utilizarán los siguientes criterios: casas donde pasa el acuse (10), Habitantes encuestado (15) intencionada de 10 familias (22 Individuos) del sector el centro Municipio Cruz Paredes.

La validez, según Sampieri (2006), en términos generales, se refiere al grado en que un instrumento realmente mide la variable que pretende medir. Por ejemplo, un instrumento válido para medir la inteligencia debe medir la inteligencia y no la memoria. Una prueba sobre conocimientos de historia tiene que medir esto y no conocimientos de literatura histórica. Un método para medir el rendimiento bursátil, tiene que medir precisamente esto y no la imagen de una empresa. En apariencia es sencillo lograr. (pág. 277). La confiabilidad de un instrumento de medición se refiere al grado en que su aplicación repetida al mismo sujeto u objeto produce resultados iguales.

\section{Resultado}

La presente investigación se apoya en la aplicación de un conjunto de estrategias que fueron claves para desarrollar la investigación y así obtener resultados reales y precisos, por medios de conversatorios, charlas, videos, talleres con carácter explicativos a lo que se refiere en el cuidado y conservación de la quebrada la Ciénaga Ubicado en el Municipio Cruz Paredes parroquia Barrancas del estado Barinas, el cual se pudo recolectar datos muy precisos sobre la situación que se está generando en dicho sector antes mencionado, donde los habitantes han destacado que el sector no cuenta con métodos, técnicas ni herramientas y entes gubernamentales 0 no gubernamentales que les suministren información sobre la problemática alarmante que está ocurriendo en la quebrada.

Proporcionándole solución y respuesta a las variables establecidas se pudo notar por medio de la observación directa la aceptación que tiene los 
habitantes del sector el centro en aplicar diferentes estrategias, métodos que sirvan para lograr el equilibrio y la preservación del ambiente, y así garantizar un ambiente sano libre de contaminante y el resguardo de los recursos naturales.

\section{Conclusiones}

Las conclusiones que han generado la siguiente investigación fueron claras y precisas la población Barranqueña no cuenta con entes activos que busquen solución e impartan información sobre el problema de la contaminación que está generando las personas, el cual trae como consecuencia la degradación de uno de los recursos naturales no renovables más importante del planeta como lo es el agua potable (dulce), sin embargo se logró llevar las estrategias pedagógicas pertinentes. Como también los diferentes tipos de herramientas necesarias, para que los pobladores del sector "El centro", Parroquia Barrancas, Municipio Cruz Paredes, Estado Barinas sean capaces de resolver los problemas que están generando la contaminación.

Por lo tanto se conviertan en expertos multiplicadores de la información y así puedan solucionar dicho problema ambiental, de tal manera que puedan gozar de un ambiente sano libre de cualquier contaminante y lo más importante asegurando un mejor futuro a nuestro niños, es importante resaltar que las estrategias empleadas fueron las correcta ya que se logró un aprendizaje significativo llegando a tocar el lado positivo de las personas dándoles ver que lo que está ocurriendo en la quebrada es alarmante por lo tanto era necesario actuar lo más pronto posible ante esta situación antes que la contaminación acabara con este cauce y de esa manera el agua que es una de las fuentes principales para el consumo de los habitante de este municipio desapareciera por completo y generara una preocupación aún más alarmante. 


\section{Recomendaciones}

Ya obteniendo la información recolectada en la investigación y siendo procesada y analizada se llega a generar unas series de recomendaciones:

- Promover el cuidado y preservación de los recursos naturales

- Realizar jornadas sensibilizadoras charlas, talleres foros entre otros sobre la preservación del ambiente

- Realizar jornadas para la recolección de desechos sólidos y disposición de los mismos.

- Llevar unos seguimientos sobre las actividades empleadas a la conservación de la quebrada.

- Realizar mesa redonda donde se discutan las resultados y beneficios en la implementación de las estrategias en la conservación de la quebrada

- Llevar los resultados y beneficios que se obtenga a otras comunidades

- Involucrar el número de entes gubernamentales y no gubernamentales en el proyecto de conservación venideros

\section{Referencias}

Cano, E. (2007). Las competencias docentes, el perfil del nuevo docente. Caracas, Venezuela. [Documento en Línea]. Recuperado de: https://books.google.co.ve/books?id=s1V7CAAAQBAJ

Dagnubys, J. (2009). Estrategia de Intervención Comunitaria para la Conservación y Mantenimiento del Rio Santa Bárbara del año 2008. Municipio Ezequiel Zamora del estado Barinas, Venezuela.

Gabriela, C. (2010). "Estrategias Pedagógicas y de Sensibilización en la Educación Ambiental". Municipio Guanare, Estado Portuguesa Venezuela. [Documento en Línea]. Recuperado de: 
http://app.vpa.unellez.edu.ve/bibliotecavpa/opac css/doc num.php?ex plnum id=11

Hernandez, E. (2011a,b). Gestión del Patrimonio Natural, Modulo 6. CCPB, Programa de Desarrollo de Capacidades para el Caribe, para el Patrimonio Mundial. La Habana: UNESCO, págs. 68. Recuperado de: http://whc.unesco.org/document/107154

León, A. (1998). Pedagogía ambiental Enciclopedia de Pedagogía / psicología (1ra. edición). P521 Alfatemática S.A. de C.V.

Ley Orgánica del Ambiente (2006). Gaceta Oficial N. 5833e. Publicada el 22 de diciembre. [Documento en Línea]. Recuperado de:

https://es.scribd.com/doc/47588555/LEY-ORGANICA-DEL-AMBIENTE Sampieri, H. (2010). Metodología de la investigación. México D.F. Mexicana, Reg. Núm. 736. [Documento en Línea]. Recuperado de:

http://www.academia.edu/6399195/Metodologia de la investigacion 5ta Edicion Sampieri

Sampieri, H. (2006). Metodología de la investigación. [Documento en Línea]. Recuperado de:

https://scholar.google.co.ve/citations?user=SI208icAAAAJ\&hl=es\&oi=s ra 


\section{Jesús Manuel Torres Martínez \\ e-mail: jesm14@hotmail.com}

Nacido en Barrancas estado Barinas cursante de

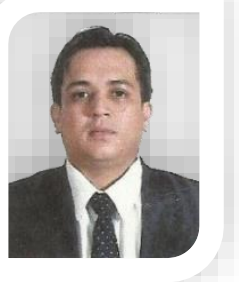
postgrado en Educación Ambiente y Desarrollo en la Universidad Experimental Libertador (UPEL), Barinas estado Barinas. Técnico medio en agropecuaria. Escuela agronómica salesiana "San José" Barinas estado Barinas (2001). Técnico Superior en Ciencias Agropecuarias del Instituto Universitario de Tecnología Agroindustrial región los Andes, San Cristóbal estado Táchira (2005). Profesor mención: educación agropecuaria Universidad Pedagógica Experimental Libertador. Gervasio rubio estado Táchira. (2009).

Actualmente se desempeña como docente de campo en maquinarias agrícola y ovinos y caprino de Escuela agronómica salesiana "san José" Barinas estado Barinas año 2010 astas 2016 Durante 2 años y medios me desempeñe como técnico de campo en el proyecto manejo integrado de plagas (MIP) en el (SASA) adscrito al ministerio de agricultura y tierra. 2006-2009 1 año como jefe de área y técnico de las áreas de cultivos de cereales y maquinarias agrícolas en la escuela agronómica salesiana barinas 2010-2011 5 Año como Técnico.; de la Asignatura Maquinaria Agrícola y Ovinos y Caprino en la escuela agronómica salesiana 2011-2016.

Como participante en más 34 jornadas en áreas de campo agrícola, nacional e internacionales al igual de congresos en la misma área. Asimismo, investigador en el área sobre la conservación de la quebrada la Ciénaga.

El contenido de este manuscrito se difunde bajo una Licencia de Creative Commons ReconocimientoNoComercial-Compartirlgual 4.0 Internacional 